\title{
Tidal evolution of stars hosting massive close-in planets
}

\author{
S. Ferraz-Mello ${ }^{1}$, L. F. R. Moda ${ }^{1}$, J. D. do Nascimento Jr. ${ }^{2}$ and \\ E. S.Pereira ${ }^{1}$ \\ ${ }^{1}$ Instituto de Astronomia, Geofísica e Ciências Atmosféricas, Universidade de São Paulo, Brasil \\ email: sylvio @ usp.br \\ ${ }^{2}$ Dep. Fisica Teórica e Experimental, Universidade Federal do Rio Grande do Norte, Natal \\ 59072-970, Brasil
}

\begin{abstract}
Close-in massive planets transfer angular momentum to their host stars and influence their rotation through the torques associated with the tides raised on the star by the planet. For a star hosting a hot Jupiter, the limit of distance below which tidal torques cannot be neglected grows from $a \sim 0.04$ to $a \sim 0.07 \mathrm{AU}$ as the mass of the planet grows from 0.5 to $4 \mathrm{M}_{\mathrm{Jup}}$.
\end{abstract}

Close-in massive planets transfer angular momentum to their host stars and influence their rotation, which will evolve differently from what it would be in absence of the closein companion. If the star is not active and is single, the rotation speed remains high, and close to its primordial state. But if a non-active star has a close-in massive companion, its rotation evolves towards period values close to the orbital period of the companion.

When the star is active, the situation is more complex. In that case, a single star loses rotation speed and its rotation period grows proportionally to the square root of the time (Skumanich law; see Skumanich, 1972). However, if it has a massive close-in companion, the star's rotation evolves under the control of two factors: (1) The stellar magnetic wind, which causes a loss of the stellar rotational angular momentum (see Bouvier, 2013); and (2) the tides, which transfer angular momentum from the orbital motion of the companion to the stellar rotation. The scenario is then the following: In the initial stage, the star has a very fast rotation resulting from the contraction of the primordial cloud from which it formed, but looses speed very fast. The rate of variation of the angular rotation velocity is proportional to the cube of its value. As the rotation decreases, the variation becomes slower up to reach a situation in which the loss of angular momentum due to the magnetic wind braking is compensated by the angular momentum tidally transferred to the star from the orbit of the companion. This tidal interaction also affects the orbital parameters of the companion, and this equilibrium situation is dynamical: the companion is slowly falling toward the star and the proximity of the two bodies enhances all tidal effects, including the acceleration of the stellar rotation. The companion may eventually fall on the star and quickly transfer its whole angular momentum to the star's rotation. From this moment on, the stellar rotation resumes its evolution as in a single star. This resetting is important because, if the rules of gyrochronology are later applied to this star seeking to determine its age, they will result into values much smaller than the actual age of the star. In general, the rules of gyrochronology used to determine the age of single stars (see Barnes 2007, Brown 2014) are not valid for one star having (or having had) a close-in massive companion.

In this communication, we present the results of some calculations of the evolution of the rotation of stars hosting massive close-in companions taking into account the joint effects of tides and magnetic braking and we discuss the limits beyond which the star's 
rotation evolution is independent of the existence of a planet around it. The experiments reported here were designed to enhance the influence of the tidal star-planet interaction on the rotation of the host stars and were limited to massive planets and to the socalled coplanar model in which the stellar rotation axis is perpendicular to the planetary orbital plane. However, the same model can be used in more extended studies dealing, for instance, with the distribution of the stellar rotational periods vs. orbital periods, which are currently available for a great number of KEPLER objects (McQuillan et al. 2013; Teitler and Königl, 2014).

In the experiments, we have used Ferraz-Mello's creep tide theory (Ferraz-Mello, 2013, 2015 ) to compute the tidal evolution of the system and the transfer of angular momentum from the orbit of the companion to the star's rotation (or vice-versa in the initial stages, when the star is spinning much faster than the companion's orbital motion), and the Bouvier et al.s formulas (Bouvier et al. 1997) for the angular momentum leakage due to the stellar wind, which reproduces fairly well the Skumanich law and the observed rotations of the stars in open clusters of several ages.

We have considered one Jupiter-like planet, initially in orbit around an active star of mass $1.1 \mathrm{M}_{\odot}$, the upper limit of the range of validity of Bouvier et al.s formulas for the leakage of angular momentum. In each experiment, we considered 4 initial (current) semimajor axes: $0.03,0.04,0.05,0.06 \mathrm{AU}$ (orbital periods equal to 1.81, 2.79, 3.89 and 5.12 days, resp.) and 3 values of the tidal relaxation factor: 20, 40 and $80 \mathrm{~s}^{-1}$. These values of the relaxation factor are the same used in the experiments reported in Ferraz-Mello et al. (2015) and are in the range of values determined there by comparing the expected evolution of the stellar rotation under the joint effect of tides and braking to its observed rotational period, for several host $\mathrm{G}$ stars of known isochronal age. These relaxation factors correspond to the quality factor $Q$ in the interval $1-6 \times 10^{6}$ (if $k_{2}=0.2$ ), which is inside the range of dissipation values determined by Hansen's (2012) from the analysis of the survival of short-period planets and of the limits for circularization of planets with highly eccentric orbits. They correspond to a less dissipative scenario than the one adopted by Jackson et al. (2008) in earlier studies.

We choose to show here the results of the experiments for one host star whose initial (current) rotation period is 10 days. These results show the same main features as the results from experiments with stars endowed of faster or slower rotation. First, it is shown that the transfer of angular momentum from the orbit always happen when $a \leqslant 0.04 \mathrm{AU}$. When $a=0.03 \mathrm{AU}$, the companion falls on the star in less than $2 \mathrm{Gyr}$. The transfer of angular momentum of the companion's orbit to the star accelerates its rotation and the period eventually decreases to much less than 10 days (see fig. 1). The falls are not always clearly visible in fig. 1 , but one may find then at the times corresponding to the end of the decrease of the stellar rotation period. At the end of the life of the companion, the stellar rotation is strongly accelerated. When $a=0.04 \mathrm{AU}$, the behavior is similar, but the remaining lifetime of the companion is much longer. When $a \geqslant 0.05 \mathrm{AU}$, the transfer of angular momentum from the orbit to the star is negligible. The corresponding curves are the envelope of the evolution tracks shown in fig. 1: the uppermost curve for $t>0$, and lowest for $t<0$. For more massive planets these limits are larger. For instance, for a $4 \mathrm{M}_{\text {Jup }}$-planet, the transfer is significant up to $a=0.06 \mathrm{AU}$.

The evolution tracks shown in fig. 1 for $t<0$ allow us to have an estimate of the age of the star. For instance, a star with the same mass $\left(1.1 \mathrm{M}_{\odot}\right)$ and current orbital period $(10 \mathrm{~d})$ and with a very close companion in circular orbit, may be expected to have an age a little less than 1 Gyr. If the companion is in an eccentric orbit, the estimated ages are spread over a larger interval. For instance, when the initial (current) eccentricity is $e=0.2$, the age of a star with the above fixed characteristics may be $\sim 1.5$ Gyr when 

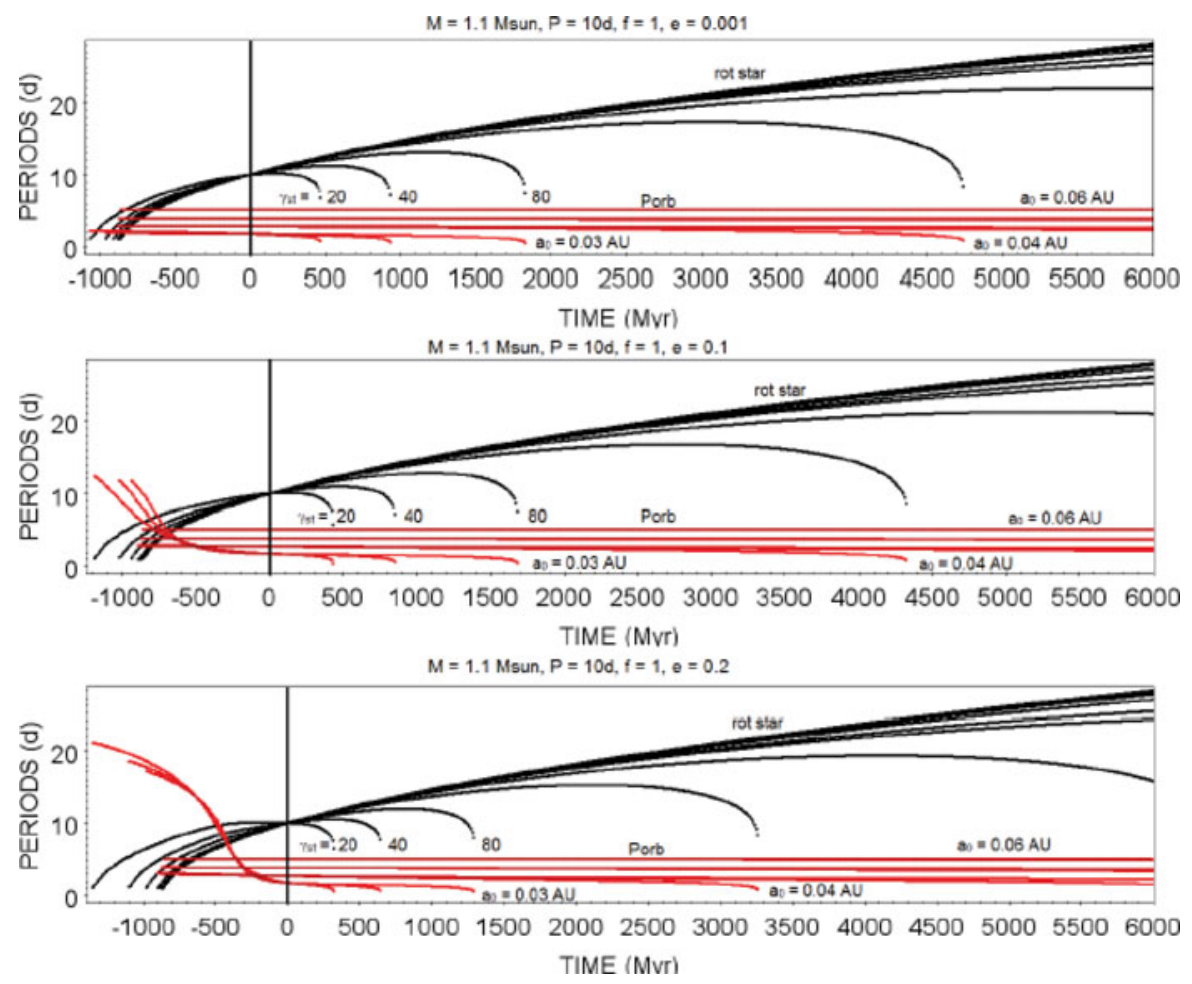

Figure 1. Evolution of the orbital period of one hot Jupiter (red) and the corresponding evolution of the rotational period of its active host star (black). Masses: $1 \mathrm{M}_{\mathrm{Jup}}$ and $1.1 \mathrm{M}_{\odot}$ resp. Initial (current) semi-major axes: 0.03 to $0.06 \mathrm{AU}$. Initial (current) eccentricities 0 (top), 0.1 (mid) and 0.2 (bottom). Initial (current) rotational period of the star: 10 days. Relaxation factors $\gamma=20,40$ and $80 \mathrm{~s}^{-1}$.

$a=0.03$ if the star is very dissipative $\left(\gamma=20 \mathrm{~s}^{-1}\right)$. If the planet is located farther away, say $a=0.04-0.05 \mathrm{AU}$, the estimate is again close to $1 \mathrm{Gyr}$.

The other curves shown in fig. 1 correspond to the orbital period of the planet which, in the conditions of the experiments done, is always decreasing. This means that, in the past, the companion is expected to have been moving in a more distant orbit. The figures shown for $t<0$ may be considered as possible past evolutionary tracks of the system. However, these tracks are critically dependent on the adopted initial (current) parameters, mainly the orbit eccentricity and the stellar rotational period. The actual evolution for a given star may have been very different.

Similar calculations were done for several values of the planetary mass showing the variation of the limit value from $a \sim 0.04$ for a hot Jupiter with mass $0.5 \mathrm{M}_{\mathrm{Jup}}$ to $a \sim 0.07 \mathrm{AU}$ for super Jupiters with mass $4 \mathrm{M}_{\mathrm{Jup}}$.

When the considered stars are not active, the behavior is much simpler because the stellar rotation drifts towards values close to the orbital period of the companion. Since these stars are generally more massive, the simulations in the absence of magnetic braking considered a host star of $1.3 \mathrm{M}_{\odot}$. Again, the initial (current) semi-major axes were fixed at $0.03,0.04,0.05,0.06 \mathrm{AU}$ (orbital periods equal to 1.66, 2.56, 3.58 and 4.71 days, resp.). Various values for the initial stellar rotational period were adopted. As before, the effects were visible only for initial semi-major axes $a \leqslant 0.04$ AU. For $a \geqslant 0.05$ AU, the stellar rotational period remains almost constant. When $a \leqslant 0.04$, the planet transfers 

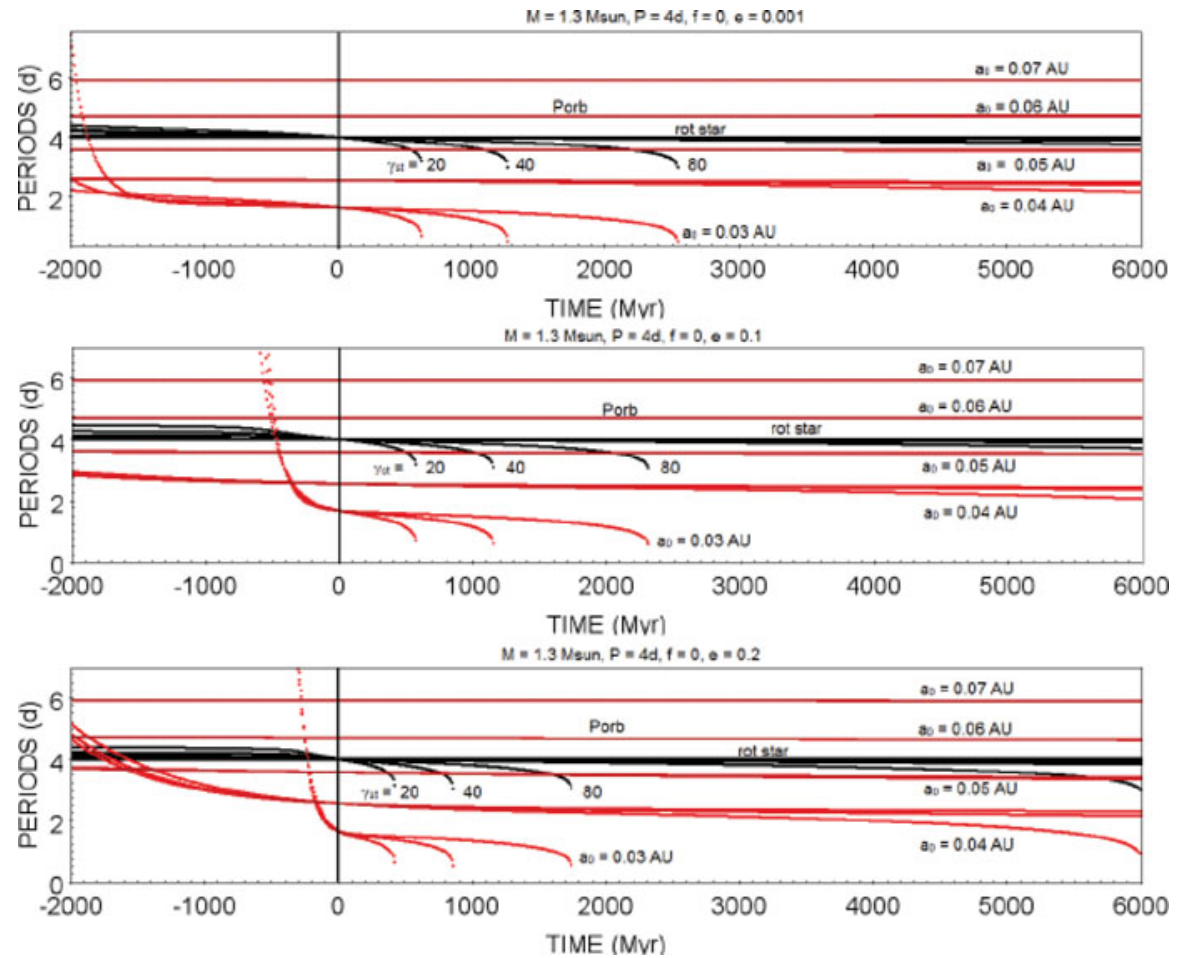

Figure 2. Same as fig. 1 but in the case of a non-active host star with mass $1.3 \mathrm{M}_{\odot}$ and initial (current) rotational period 4 days.

angular momentum to the rotation of the star, accelerating it. The star's rotation period decreases monotonically. The companion eventually falls on the star.

Fig. 2 shows the evolution of the orbital period of the hot Jupiter and the rotational period of a non active star whose current period is 4 days. Larger periods are not shown. In fact, the rotations measured by the space telescope KEPLER of the stars in the clusters NGC 6811 and NGC 6815 (Meibom et al., 2011, 2015) show that stars in this mass range have their rotational periods limited to a few days, even for ages as large as 2.5 Gyr.

We have used here the same tidal relaxation values used for solar-like stars $(20,40$ and $80 \mathrm{~s}^{-1}$ ) but it is worth recalling that dissipation in F stars is not as well constrained as in $\mathrm{G}$ stars and larger values of $\gamma$ may be possible (Barker and Ogilvie, 2009). The technique used to determine the tidal relaxation factor in active stars with $m \leqslant 1.1 \mathrm{M}_{\odot}$ cannot be used for the non-active stars because all well observed F stars with massive close-in companions have the rotation period almost equal to the orbital period, within the standard errors of their determinations, making impossible an estimation of the past evolution of the stellar rotation for different relaxation factors. The only exception is CoRoT-3 (Ferraz-Mello, in prep.). In this case the faster stellar rotation and the strong interaction between the star and the companion, a brown dwarf, allowed us to constrain the stellar tidal relaxation factor to the interval $40-70 \mathrm{~s}^{-1}$, which corresponds, for this star, to $Q \sim 6-9 \times 10^{6}$ (for $k_{2}=0.2$ ). This result agrees with Barker and Ogilvie (2009) statement that in lower mass F stars, the dissipation is similar but slightly weaker than that of solar-type stars.

Finally, for the sake of completeness, fig. 3 shows the variation of the eccentricity in the two non-circular cases of fig. 1 (In the cases of fig.2, the eccentricity variation is similar). 

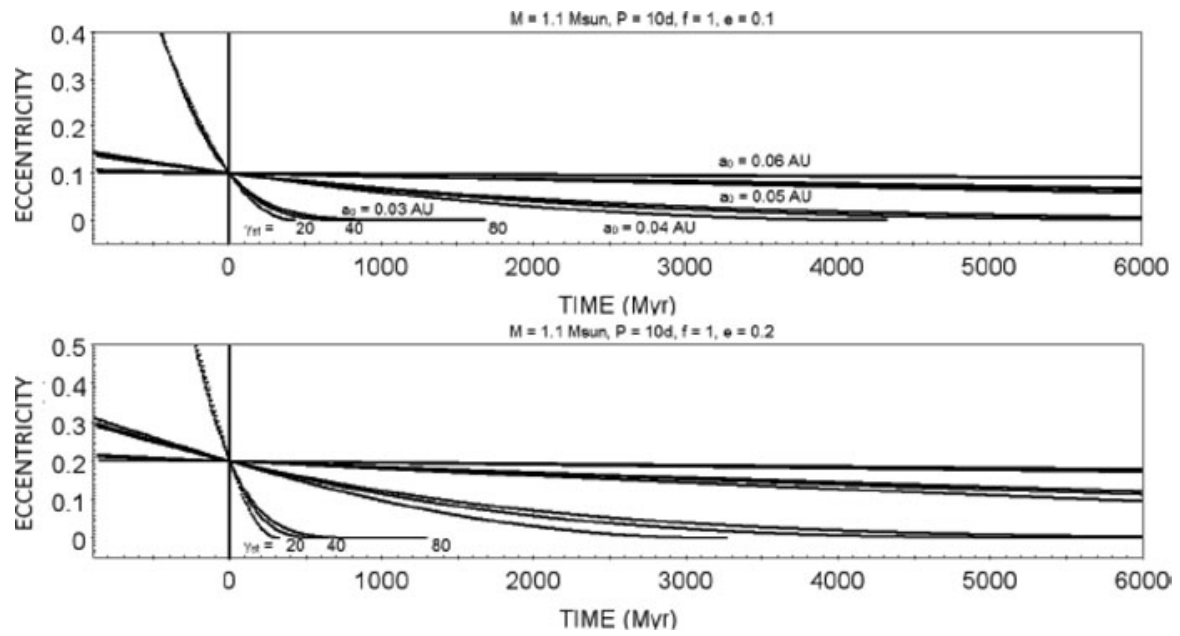

Figure 3. Evolution of the orbital eccentricity of the hot Jupiter in the solutions shown in fig. 1 for the initial (current) eccentricities 0.1 (top) and 0.2 (bottom).

This figure shows the tendency to circularization of all cases, which is as more important as the planet is closer to the star. The projection to the past when $a=0.03$ leads to very high eccentricities indicating that we should not expect to find eccentric systems in the case of too close planets. When the current system is already circularized, it is not possible to make an estimation of the past evolution of the eccentricity, because it is not possible to know when the planet reached the circular orbit.

\section{Conclusion}

We reported in this communication some results of an extended series of calculations of the tidal evolution of planetary systems, in which the companion is a massive close-in planet. The results extend similar ones published in Ferraz-Mello et al. (2015) and allow to evaluate the limits beyond which the consideration of tidal effects cannot be neglected since they influence both the evolution of the planet orbit and the host star rotation. For systems with Jupiter-like planets, the rules of gyrochronology used for single stars can only be applied when the semi-major axis of the Jupiter-like planet is larger than a given limit. The reported simulations indicate that this limit grows from $a \sim 0.04$ to $a \sim 0.07$ $\mathrm{AU}$ as the mass of the planet grows from 0.5 to $4 \mathrm{M}_{\mathrm{Jup}}$. Comparing these values with the data of the known exoplanets, we see that about 200 of the currently known transiting hot Jupiters and brown dwarfs may affect significantly the rotation of their host stars.

\section{Acknowledgement}

This investigation was supported by grants CNPq 306146/2010-0, FAPESP 2014/134074 and 2015/15154-9 and by INCT Inespaço procs. FAPESP 2008/57866-1 and CNPq $574004 / 2008-4$.

\section{References}

Barker, A. J. \& Ogilvie, G. I. 2009. MNRAS 395, 2268

Barnes, S. A. 2007, ApJ, 669 id. 1167

Bouvier, J., Forestini, M., \& Allain, S. 1997 A $\& A$, 326, 1023

Bouvier, J. 2013, EAS Publ. Series Vol. 62, 143

Brown, D. J. A. 2014, MNRAS 442, 1844 
Ferraz-Mello, S. 2013, Celest. Mech. Dyn. Astr. 116, 109-140 (arXiv: 1204.3957)

Ferraz-Mello, S. 2015, Celest. Mech. Dyn. Astr. 122, 359-389 (arXiv: 1505.05384)

Ferraz-Mello, S., Santos, M., Folonier, H., Csizmadia, S., Nascimento Jr, J. D. D., \& Pätzold, M. 2015 ApJ 807, id. 78 (arXiv: 1503.04369).

Hansen, B. M. S. 2012. ApJ 757: 6

Jackson, B., Greenberg, R., \& Barnes, R. 2008. ApJ 678, 1396

McQuillan, A., Mazeh, T., \& Aigrain, S. 2013. ApJ Letters, 775, L11.

Meibom, S., Barnes, S. A., Latham, D. W. et al. 2011, ApJ Letters, 733, L9

Meibom, S., Barnes, S. A., Platais, I. et al. 2015 Nature 517, 589

Skumanich, A. 1972, ApJ, 171, 565

Teitler, S. \& Königl, A., 2014. ApJ 786: 139 